\title{
SUMMARY OF DISCUSSION ON INDIVIDUAL OBJECTS
}

\author{
Discussion chairman Scott Kenyon \\ Discussion recorded by David Allen
}

\section{HIGH VELOCITY WINDS FROM THE HOT COMPONENT}

A number of contributors had suggested the existence of high-velocity winds which presumably originates in the hot components. One possible piece of evidence was broad wings to Ly $\alpha$. Kenyon opened the discussion by asking whether these wings indeed arose in a wind or merely reflected scattering processes. The absence of broad wings on other lines, Kwok noted, does not disprove a wind. Viotti did, however, caution that Ly $\alpha$ will be self-absorbed and have scattering wings, so that it is not the best choice. Furthermore, as Selvelli added, even isolated M giants show broad wings to Ly $\alpha$ (Johanssen \& Jordan, 1986, Mon. Not. R. astr. Soc., 210, 239) with a redward shift of about $1 \AA$, as seen in symbiotic stars. Schmid pointed out that Ly $\alpha$ could be scattered in a high-velocity neutral region illuminated by the ionized gas.

Turning to the anomalous C IV doublet ratios, Allen noted that a wind that was sufficiently optically thick to create a photosphere could explain the correlations seen in RX Pup and Z And between the $\lambda 1548 / \lambda 1550$ ratio and the continuum brightness. Michalitsianos felt that there was a sound theoretical understanding of the effect of the wind on the doublet ratio, giving it the potential to be a good diagnostic of the wind. However, very few stars are bright enough to be studied with IUE.

\section{BIPOLAR STRUCTURES}

Kenyon steered the discussion to the radio morphologies. The $\mathrm{CH}$ Cygni radio jet suggested collimated outflow at high velocities. Seaquist reiterated that the data require expansion rather than the progressive ionization of pre-existing features, and asked whether a disk could produce the collimation. Slovak, noting the absence of a strong magnetic field to serve as a collimator, drew parallels with the T Tauri stars. In the symbiotic novae however, as Kenyon pointed out, little or no disk is likely to form from the wind: why do the radio observations still suggest bipolarity? Chochol proposed the formation of an excretion disk around the hot star, but this was not widely accepted as physically viable due to problems with conservation of angular momentum.

Rao conjectured that magnetic fields too weak to detect could be relevant, particularly in the presence of differential rotation. Webbink performed a backof-envelope calculation to infer that fields of order 1 gauss were adequate. Livio 
noted that fewer than $20 \%$ of planetary nebulae are spherical whereas there is little evidence that novae are bipolar (Friedjung challenged the latter claim), so that the cool star may be responsible. The SiO masers in VX Sgr have a bipolar distribution, Stencel added, thanks to a surface field of a few tens of Gauss.

The possibility was aired that the morphology is more apparent than real. Laminar sheets seen edge-on can appear bipolar, Slovak noted, but Friedjung reckoned an unacceptable proportion would have to be seen in this way. Both Viotti and Allen questioned whether we are merely recording two components, perhaps associated with the two stars. Viotti showed a radio map of $\alpha$ Sco in which this was exactly the situation.

\section{ORBITAL PARAMETERS}

Liebowitz raised a specific problem with the quoted mass ratio in AG Peg, which did not permit acceptable masses for the stars. Slovak felt that this was a case of underestimating the errors in the original determination by Cowley \& Stencel (1973, Astrophys. J., 184, 687), and Webbink added that the velocity curves had not been corrected for reflection effects.

Turning to eccentricities, Liebowitz noted that Garcia's orbital data suggested small values, in contrast to non-interacting binaries of comparable orbital periods. Nussbaumer added that an attempt to explain V1329 Cyg using a large eccentricity had failed to work out. Webbink expected very small eccentricities among the symbiotics because the circularisation timescale is shorter than the evolution timescale for the stars in question. A thorn in the side of this argument, he admitted, is the existence of the barium stars (G-K giant plus probable white dwarf) which have $\bar{e} \sim 0.2$, and so have managed to interact and retain finite eccentricities. 